\title{
La transmisión de las desigualdades en el estado de salud: Efectos sobre la equidad intergeneracional a partir de la acumulación de capital humano
}

\author{
Berta Rivera \\ Escuela Gallega de Administración Sanitaria \\ Luis Currais \\ Paolo Rungo \\ Departamento de Análisis Económico y Administración de Empresas. \\ Universidad de A Coruña.
}

\section{Resumen}

Este trabajo analiza el papel de la salud como un factor transmisible entre generaciones y sus efectos sobre la acumulación de capital humano y la eficiencia del proceso educativo. Un efecto positivo y significativo, en este sentido, apoya la hipótesis de que el estado de salud individual contribuye a la transmisión intergeneracional de los ingresos, dado que los hijos de padres pobres tienen una mayor tendencia a la enfermedad y acumulan menos capital humano, perpetuando el círculo vicioso de la pobreza. El análisis se realiza a partir de la encuesta brasileña de hábitos de vida que permite establecer relaciones intergeneracionales de un extenso conjunto de variables. La metodología utilizada se basa en la estimación de diferentes especificaciones de acuerdo con la distribución de las variables utilizadas.

Palabras clave: Transmisión de la salud, desempeño educativo, capital humano, equidad intergeneracional.

Clasificación JEL: I10, J24, O40

\begin{abstract}
This paper analyses the role of health status as a transmissible factor across generations of the same family, and its effects on the accumulation of human capital and the efficiency of the educational process. A significant and positive effect of health on human capital formation implies that individual health affects the intergenerational transmission of income, provided that children of poor parents are more likely to get ill and they accumulate a lower level of human capital, perpetuating thus the vicious cycle of deprivation. The analysis relies on a Brazilian household survey, which permits to establish intergenerational relationships for an extended set of variables. Regarding methodology, different models have been estimated according to the distribution function of the variables been considered.
\end{abstract}

Key words: Transmission of health status, educational adequacy, human capital, intergenerational equity.

JEL Classification: I10, J24, O40 


\section{Introducción}

A partir de Becker y Tomes (1986), el análisis de la movilidad intergeneracional ha sido un tema muy discutido tanto en la literatura empírica como en la teórica. El grado de transmisión de la capacidad de generar ingresos resulta muy importante para entender fenómenos como la persistencia de la pobreza. Estudios históricos y macroeconómicos han determinado los fuertes efectos de la nutrición y la salud sobre los ingresos de largo plazo y el crecimiento económico. En este sentido, podemos encontrar trabajos empíricos recientes que han demostrado que la transmisión intergeneracional del estado de salud es un factor clave para explicar trampas intergeneracionales de pobreza.

Estos resultados presuponen dos hipótesis que merecen ser explicitadas. En primer lugar, se supone que el estado de salud es un factor transmisible entre generaciones. Currie y Moretti (2005), por ejemplo, utilizando una base de datos confidencial que incluye todos los nacimientos en California en los periodos 1960-1974 y 19822001 , encuentran que la probabilidad de nacer con bajo peso es $50 \%$ mas alta si la madre ha nacido con bajo peso ${ }^{1}$. Además, encuentran un efecto independiente y positivo del estado socioeconómico de la familia de origen: la transmisión intergeneracional del nacimiento con bajo peso es más relevante en el caso de madres pobres. Este último resultado pone en evidencia que la herencia de un determinado estado de salud puede estar determinada por las condiciones económicas de la familia; efectivamente, parece intuitivo que el estado socioeconómico de los padres tenga un fuerte impacto sobre la salud de los hijos, a través, por ejemplo, de la disponibilidad de recursos dedicados a la nutrición o la posibilidad de una adecuada asistencia médica. La existencia de una relación positiva entre ingresos familiares y salud de los niños ha sido confirmada, entre otros, por Case et al. $(2005,2002)$.

La segunda hipótesis detrás de la relación entre salud y transmisión de ingresos está relacionada con la influencia de la salud en edades tempranas sobre los ingresos durante la madurez. Una relación directa entre estas variables se puede encontrar en la productividad relativamente inferior de los trabajadores dotados de un pobre estado de salud. Muchos estudios confirman esta idea, como Beherman (1993), Beherman y Deolalikar (1998), Case et al. (2005), Currais y Rivera (2005) y Sahan y Alderman (1998), entre otros.

Es posible identificar otro mecanismo que permite explicar el papel de la salud en la transmisión de ingresos, es decir, la relación causal entre estado físico y acumulación de capital humano intelectual. Alderman et al. (2001), Glewwe et al. (2001), the Micronutrient Initiative and United Nations Children's Fund (2004), Miguel (2005), World Food Programme (2006), demuestran la existencia de una relación causal positiva entre distintos indicadores de salud, frecuencia a la escuela, capacidades cognitivas y logros académicos. Una implicación de estos estudios es que niños con un buen estado

El nacimiento con bajo peso es un buen indicador del estrado de salud en edad adulta (Currie y Moretti, 2005). 
de salud tienden a obtener mayores beneficios del proceso educativo, ceteris paribus. Al contrario, un niño dotado de un estado de salud no favorable consigue acumular un nivel mas bajo de capital humano.

El presente trabajo analiza la relación entre estado físico y educación, para entender el papel de la salud en la transmisión intergeneracional de la capacidad de generar ingresos. Así, se estudia la influencia del estado de salud sobre la acumulación de capital humano y sobre la eficiencia del proceso educativo. El trabajo esta organizado según la siguiente estructura. La sección 2 presenta las características principales de la encuesta utilizada y un análisis descriptivo de las variables de interés. La sección 3 presenta el modelo a estimar y los resultados de los ejercicios empíricos. En primer lugar, se considera la transmisión intergeneracional de la salud para analizar, a continuación, los efectos de la salud sobre la acumulación de capital educativo. La última sección resume las conclusiones principales.

\section{Los Datos}

En el análisis empírico se han utilizado los datos de la «Pesquisa sobre Padroes de Vida» (PPV), una encuesta llevada a cabo entre el 1996 y 1997 por el Istituto Brasileño de Geografía y Estadística en asociación con el Banco Mundial (IBGE, 2003). La encuesta recoge informaciones sobre 19.409 individuos y 4.800 familias, representativas de las regiones nordeste y sureste de Brasil ${ }^{2}$. Se ha elegido esta encuesta realizada en Brasil ya que tiene la ventaja de incluir tanto un extenso conjunto de variables relacionadas con el estado de salud individual (tanto indicadores objetivos como sujetivos), como indicadores históricos de los resultados del proceso educativo. Además, incluye un gran número de variables socioeconómicas. Otra característica importante, de gran utilidad para los estudios intergeneracionales, es la posibilidad de relacionar padres e hijos gracias al hecho de que todos los miembros de cada familia fueron entrevistados.

Con el fin de obtener una base de datos útil para este análisis, se han relacionado todos los individuos de cada familia, utilizando los hijos como referencia. Como resultado, se han extraído los datos de 3.440 hijos, para los cuales existe información completa sobre sus padres. En otras palabras, las 3.440 observaciones utilizadas en el análisis incluyen hijos que han sido entrevistados y cuyos padres también han sido entrevistados. Los hijos se definen como individuos con edad comprendida entre los 8 y los 30 años, que viven con sus padres. La utilización de esta definición se debe a la posibilidad de comparación con resultados de otros análisis, elegida, básicamente, por la literatura porque recoge los primeros períodos aprovechables de la enseñanza obligatoria (que habitualmente empieza a los 6 años de edad) y los mayores niveles de educación adquiridos, asimilables a estudios de postdoctorado.

2 Dada su configuración de investigación piloto, se decidió que la encuesta cubriera solo estas dos regiones de Brasil. 


\subsection{Variables de interés}

Las variables utilizadas en el análisis empírico, y los estadísticos descriptivos principales, se presentan en la Tabla 1. Se han utilizado indicadores del estado de salud objetivos y subjetivos. El índice de masa corporal (BMI) se define como la ratio entre peso y altura al cuadrado; hay que observar que las mediciones fueron llevadas a cabo por los entrevistadores. La autovaloración del estado de salud (SAHS) es una variable discreta con valores comprendidos entre 1 y 5 , en donde 1 representa la autovaloración del estado de salud mejor (excelente), y 5 la peor (muy pobre-ruim). Las variables GHEALTH (buena salud) y PHEALTH (mala salud) siguen de las respuestas de autovaloración. Su valor es igual a 1 si el individuo valora su estado de salud como excelente o muy bueno (valores 1 o 2 en SAHS) o un estado de salud pobre o muy pobre (valores 4 o 5 en SAHS) respectivamente, y cero en los otros casos. De las respuestas a los cuestionarios se pueden obtener otros indicadores útiles. La variable PROBLEMS sigue de la respuesta sobre la ocurrencia de problemas de salud durante los treinta días anteriores a la encuesta. Además, los individuos reportan si sufren de enfermedades crónicas (variable CHRONIC).

El nivel educativo ha sido medido a través de variables que tienen en cuenta tanto el nivel máximo de educación conseguido, como el tiempo para lograrlo. En particular, la variable utilizada para medir el nivel de capital humano intelectual, EAC (desempeño educativo), se define como la ratio entre el número de años de educación completados con éxito y el número de años que el individuo podría haber completado, dada su edad. Este último valor se obtiene como diferencia entre la edad del estudiante y la edad mínima obligatoria de comienzo del proceso educativo en Brasil. Hay que observar que para un individuo que podría haber completado el máximo número de años de educación, esta variable mide el nivel de capital humano intelectual, en una escala continua de 0 a 1 . Sin embargo, para los niños en edad escolar, la variable EAC se interpreta como un indicador del capital humano potencial. Explícitamente, EAC no mide directamente el nivel de capital humano, sino que es un indicador indirecto de la capacidad de los estudiantes de completar con éxito los años de estudios que componen el proceso educativo. La misma variable utilizada para los padres (EDUM y EDUF, educación de madres y padres, respectivamente), como se observa anteriormente, mide el nivel de capital humano con un valor comprendido entre 0 y 1 . La edad a la cual los hijos dejan la escuela para incorporarse en el mercado de trabajo (AGEW) se ha utilizado como proxy del tiempo dedicado a la educación.

En el análisis se utilizan otras variables de control. En particular, como características individuales, se han utilizado la edad (AGE), grupo racial (WHITE), y sexo (FEMALE, MALE). Los ingresos familiares totales (LOGRENDOM) y la localización geográfica (NORTHEAST, URBAN), caracterizan las familias. 
TABLA 1

VARIABLES Y PRINCIPALES ESTADÍSTICOS DESCRIPTIVOS

\begin{tabular}{|c|c|c|c|c|}
\hline Variable & Descripción & Hijos & Madres & Padres \\
\hline \multicolumn{2}{|c|}{ Indicador de Estado de Salud } & \multicolumn{3}{|c|}{ Media (Desv. Típica) } \\
\hline \multirow{6}{*}{$\begin{array}{l}\text { BMI } \\
\text { SAHS }\end{array}$} & Índice masa corporal & $20,04(3,86)$ & $25,61(4,97)$ & $25,17(0,15)$ \\
\hline & $1=$ excelente & $24,80 \%$ & $11,31 \%$ & $14,53 \%$ \\
\hline & 2 = muy buena & $31,42 \%$ & $20,09 \%$ & $22,12 \%$ \\
\hline & $3=$ buena & $37,09 \%$ & $38,34 \%$ & $34,59 \%$ \\
\hline & $4=$ regular & $6,22 \%$ & $27,06 \%$ & $24,51 \%$ \\
\hline & $\begin{array}{l}5=\text { pobre } \\
1=\text { valores } 1 \text { ó } 2 \text { en SAHS }\end{array}$ & $\begin{array}{l}\mathbf{0 , 0 5 \%} \% \\
\mathbf{0 . 5 6 2 2}\end{array}$ & $\begin{array}{c}3,20 \% \\
31,40 \%\end{array}$ & $\begin{array}{c}4,04 \% \\
\mathbf{3 6 , 6 5 \%}\end{array}$ \\
\hline PHEALTH & $\begin{array}{l}0=\text { en otro caso } \\
1=\text { valores } 405 \text { en SAHS, }\end{array}$ & 0,0627 & $30,26 \%$ & $28,55 \%$ \\
\hline & $0=$ en otro caso & & & \\
\hline PROBLEMS & $\begin{array}{l}1 \text { = si manifiesta problemas de salud } \\
\text { En los } 30 \text { días previos a la entrevista, } \\
0 \text { = en otro caso }\end{array}$ & 0,1939 & $24,42 \%$ & $21,34 \%$ \\
\hline CHRONIC & $\begin{array}{l}1 \text { = si padece enfermedad crónica, } \\
0=\text { en otro caso }\end{array}$ & 0,0762 & $25,99 \%$ & $22,73 \%$ \\
\hline \multicolumn{5}{|l|}{ Educación } \\
\hline $\begin{array}{l}\text { EAC } \\
\text { EDUM }\end{array}$ & $\begin{array}{l}\text { Desempeño educativo hijos } \\
\text { Desempeño educativo madres }\end{array}$ & $0,59(0,25)$ & $0,23(0,16)$ & \\
\hline EDUF & Desempeño educativo padres & & & $0,24(0,17)$ \\
\hline PUBSCHOOL & $\begin{array}{l}1=\text { si el individuo asiste a una } \\
\text { escuela pública, } 0=\text { en otro caso }\end{array}$ & $79,27 \%$ & & \\
\hline EDU1P & $\begin{array}{l}1 \text { = padre sin grado de educación, } \\
0=\text { en otro caso }\end{array}$ & & $9 \%$ & $8,83 \%$ \\
\hline EDU2P & $\begin{array}{l}1 \text { = padre con educación primaria } \\
0=\text { en otro caso }\end{array}$ & & $72,96 \%$ & $72,69 \%$ \\
\hline EDU3P & 1 = padre con educación secundaria. & & $14,39 \%$ & $14,77 \%$ \\
\hline EDU4P & $\begin{array}{l}1 \text { = padre con educación superior } \\
0=\text { en otro caso }\end{array}$ & & $3,66 \%$ & $3,72 \%$ \\
\hline AGEW & Edad de comienzo de actividad & $14,70(3,99)$ & & \\
\hline \multicolumn{5}{|c|}{ Otras características individuales } \\
\hline $\begin{array}{l}\text { AGE } \\
\text { WHITE }\end{array}$ & $\begin{array}{l}\text { Edad en años } \\
1=\text { si el individuo es blanco } \\
0=\text { en otro caso }\end{array}$ & $\begin{array}{c}15,81(5,51) \\
49,77 \%\end{array}$ & & \\
\hline FEMALE & $\begin{array}{l}1=\text { si es mujer } \\
0=\text { en otro caso }\end{array}$ & $46,63 \%$ & & \\
\hline MALE & $\begin{array}{l}1=\text { si es hombre } \\
0=\text { en otro caso }\end{array}$ & $53,37 \%$ & & \\
\hline \multicolumn{5}{|l|}{ Hogar } \\
\hline $\begin{array}{l}\text { LOGRENDOM } \\
\text { URBAN }\end{array}$ & $\begin{array}{l}\text { Log de la renta total familiar } \\
1=\text { si la familia vive en área urbana } \\
0=\text { en otro caso }\end{array}$ & $\begin{array}{l}7,07(1,01) \\
80,47 \%\end{array}$ & & \\
\hline NORTHEAST & $\begin{array}{l}1=\text { si la familia vive en un área } \\
\text { del nordeste, } 0=\text { en otro caso }\end{array}$ & $49,51 \%$ & & \\
\hline
\end{tabular}




\subsection{Análisis descriptivo}

En la Figura 1 observamos la distribución del estado de salud de los hijos condicionado al estado de salud de los padres. Así, se considera la autovaloración del estado de salud, y se presentan los valores de la variable SAHS para los hijos, por cada valor de la misma variable para madres y padres. Es evidente la relación directa entre estado de salud de padres e hijos, en particular por niveles altos de autovaloración de la salud. A medida que la salud de los padres empeora esta influencia se concentra más en valores centrales de la variable, aunque no deja de ser relevante el empeoramiento de la autovaloración del estado de salud de los hijos. Del cálculo de los valores medios y de las varianzas para cada caso se deduce que la relación entre salud de la madre y salud de los hijos es más fuerte respecto a la relación entre padres e hijos. Esta circunstancia es coherente con la idea que madres e hijos tienen una relación física más directa, debido al estado de embarazo.

En la Figura 2 se presentan las distribuciones de la variable SAHS para los hijos, para intervalos seleccionados de la variable EAC. Recordemos que esta variable mide la relación entre el número de años de educación completados y el número de años que el encuestado podría haber completado dada su edad. En particular, los extremos de los intervalos considerados corresponden a los percentiles $(0,25,50,100)$. Se puede observar la existencia de una relación positiva entre estado de salud de los hijos y su educación. A medida que avanzamos hacia niveles educativos superiores mejora el estado de salud autodeclarado, principalmente en la primera categoría considerada como de salud excelente.

\section{Estimaciones y resultados}

\subsection{Modelo a estimar}

La existencia de una relación causal positiva entre capital humano de los padres y capital humano de los hijos constituye el punto de departida del presente trabajo,

$$
e_{1 t}=\beta e_{0 i}+\varepsilon_{t},
$$

dónde $e_{1 t}\left(e_{0 t}\right)$ es un indicador del nivel educativo del hijo (de los padres) y $\varepsilon$ es un término de error específico para cada individuo, distribuido normalmente. La ecuación 1 pretende medir la influencia directa de la educación de los padres sobre la educación de sus hijos. Hay que observar, sin embargo, que la educación de los padres puede ejercer su influencia también a través del canal de la salud. Un buen nivel educativo de los padres (y, en consecuencia, un nivel alto de renta) puede estar en la base de un estado de salud favorable de los hijos. En consecuencia, si la salud influye en las capacidades de aprendizaje de los niños, como se apunta en la introducción, parte del efecto de la educación de los padres sobre la de los hijos se podría explicar a través de esta vía secundaria. 
FIGURA 1

DISTRIBUCIÓN DE SAHS PARA LOS HIJOS, SEGÚN VALOR DE SAHS PARA LOS PADRES

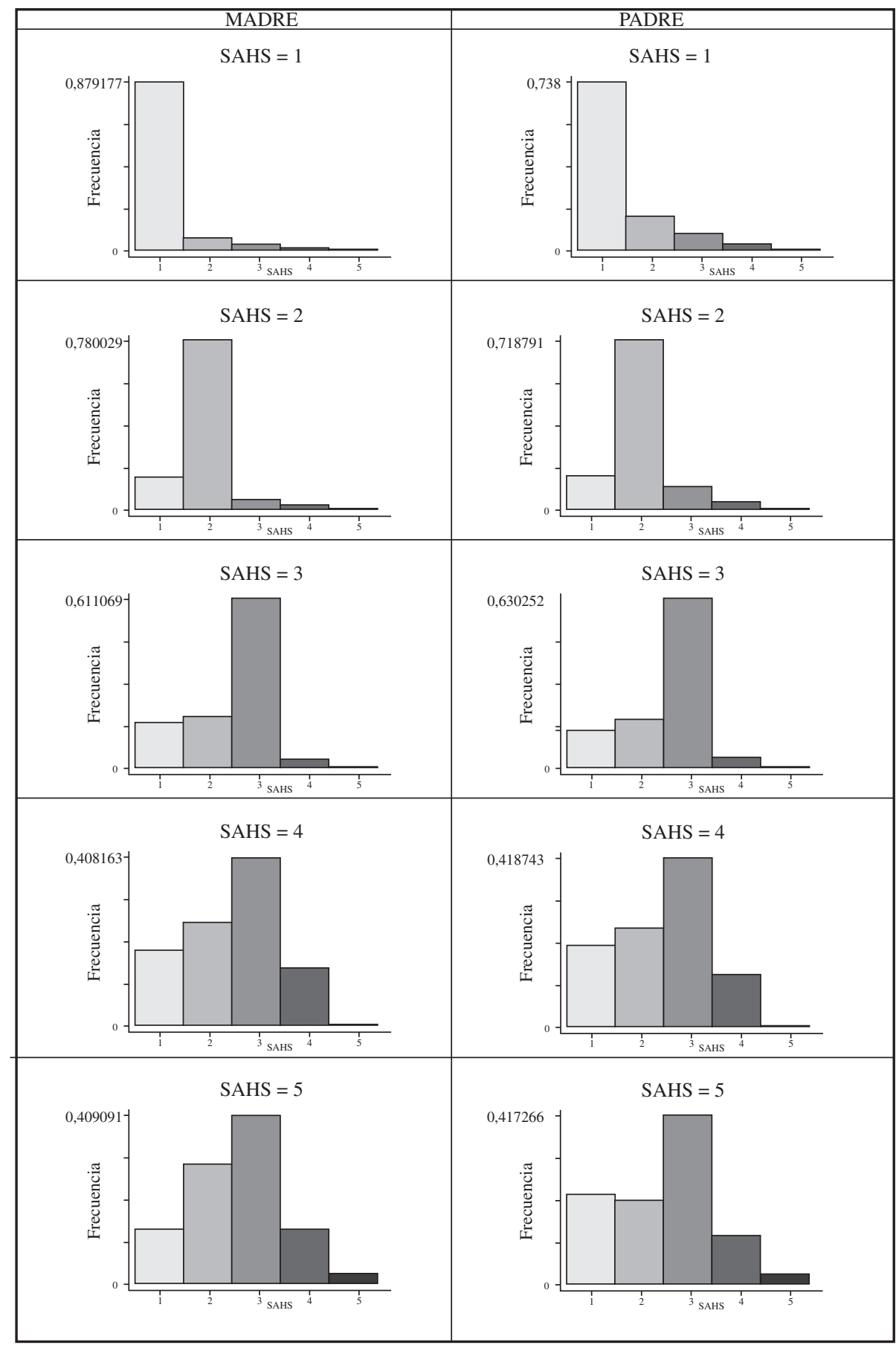


FIGURA 2

DISTRIBUCIÓN DE SAHS PARA LOS HIJOS, SEGÚN SU NIVEL DE EDUCACIÓN (INTERVALOS SELECCIONADOS A PARTIR DE LA FUNCIÓN DE DISTRIBUCIÓN DE EAC)

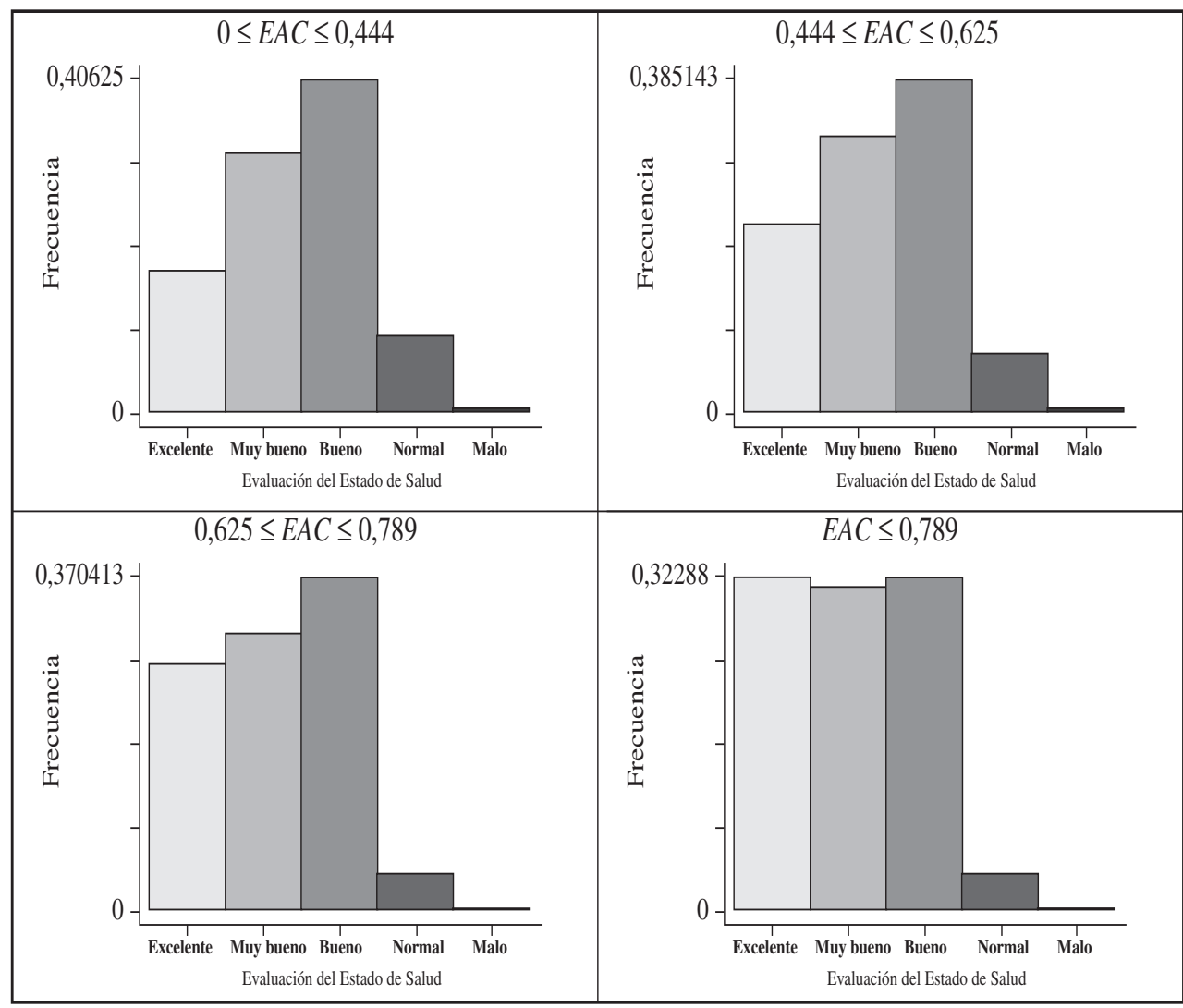

Asimismo, hay que tener en cuenta un efecto directo de la salud de los padres (en particular, de la madre) sobre la salud de los hijos, parcialmente debido a causas genéticas (e.g. Currie y Moretti, 2005). La transmisión directa del estado de salud también genera consecuencias sobre la acumulación de capital humano intelectual, por las mismas causas antes expuestas. En el siguiente sistema se consideran estas relaciones,

$$
\begin{aligned}
& H_{1 t}=\delta H_{0 t}+\gamma e_{0 t}+\varepsilon_{t}^{2} \\
& e_{1 t}=\alpha H_{1 t}+\beta_{1} e_{0 t}+\varepsilon_{t}^{1}
\end{aligned}
$$

La ecuación (2) expresa el estado de salud de los hijos, $H_{1 t}$, como función de la salud $H_{0 t}$ y de la educación de sus padres. La ecuación (3) incluye el estado de salud 
como variable explicativa de la educación de los hijos. Sustituyendo (2) en (3), se obtiene la educación de los hijos como función del capital humano de los padres, en términos de salud y educación,

$$
e_{1 t}=\phi H_{0 t}+\phi_{1} e_{0 t}+u_{t}
$$

donde $\phi=\alpha \delta, \phi_{1}=\alpha \gamma+\beta_{1}$ y $u_{1}=\alpha \varepsilon^{2}+\varepsilon_{t}^{1}$. La ecuación (4) pone en evidencia que el efecto de la educación de los padres sobre la educación de los hijos depende también de su influencia sobre el estado de salud. A partir de la ecuación 1, se pretende analizar la transmisión intergeneracional del estado de salud (ecuación 2). Si se verifica esta relación, y si los coeficiente estimados de (3) tienen signos positivos, entonces se puede concluir que la transmisión de capital humano intelectual depende tanto del nivel educativo como del estado de salud de la familia de origen (4).

\subsection{La influencia del estado de salud de los padres sobre el estado de salud de sus hijos}

En primer lugar analizamos la transmisión intergeneracional del estado de salud a través de la estimación de la ecuación (2) considerada anteriormente. Los resultados obtenidos se presentan en la Tabla 2. Se han utilizado distintos indicadores de salud como variables dependientes. Los mismos indicadores, referidos a los padres, constituyen las variables explicativas del modelo. La metodología utilizada en los ejercicios empíricos varía según la función de distribución de las variables dependientes. La columna 1 ilustra los resultados de una regresión en la cual la variable dependiente es el BMI de los niños y, por lo tanto, se han utilizado Mínimos Cuadrados. En las columnas de 2 a 4 se presentan los resultados de la estimación de modelos probit. Finalmente, se ha estimado un modelo probit ordenado cuando la variable dependiente es la autovaloración del estado de salud. Todos los modelos considerados permiten concluir que el estado de salud de los padres afecta positivamente la salud de los hijos. Además, el uso de indicadores objetivos o subjetivos del estado de salud no altera el resultado de una influencia mayor de la salud de las madres sobre la salud de los hijos.

Por lo que respecta al resto de variables los resultados se encuentra dentro de lo esperado. Un mayor nivel de renta presenta un efecto positivo sobre el estado de salud al igual que mayores niveles educativos de la madre o una mayor edad (debemos recordar que el tramo de edad de la muestra de referencia es de 8 a 30 años). El efecto contrario se encuentra cuando el lugar de residencia del individuo es el nordeste de Brasil, que corresponde con una de las zonas más pobres del país. Ser mujer también determina un peor estado de salud declarado como es habitual en este tipo de estudios.

En base a estos resultados parece existir una significativa relación causal entre capital humano de padres e hijos al evidenciarse una influencia directa del estado de salud de los padres sobre los hijos. La transmisión del estado de salud, en particular, ge- 
TABLA 2

EFECTOS DE LA SALUD DE LOS PADRES SOBRE LA SALUD DE LOS HIJOS

\begin{tabular}{|c|c|c|c|c|c|}
\hline $\begin{array}{l}\text { Variable Dependiente } \\
\text { Variables }\end{array}$ & BMI & PROBLEM & GHEALTH & CHRONIC & SAHS \\
\hline $\begin{array}{l}\text { Salud Madre } \\
\text { Salud Padre }\end{array}$ & $\begin{array}{l}\mathbf{0 , 1 4 *} \\
(9,60) \\
\mathbf{0 , 1 2 *} \\
(6,56)\end{array}$ & $\begin{array}{c}\mathbf{0 , 6 8 *} \\
(11,27) \\
\mathbf{0 , 4 0 *} \\
(6,24)\end{array}$ & $\begin{array}{c}\mathbf{1 , 3 3 *} \\
(16,85) \\
\mathbf{0 , 8 7 *} \\
(13,05)\end{array}$ & $\begin{array}{l}\mathbf{0 , 3 4 *} \\
(4,69) \\
\mathbf{0 , 3 2 *} \\
(4,05)\end{array}$ & $\begin{array}{c}\mathbf{0 , 3 7 *} \\
(15,21) \\
\mathbf{0 , 2 2 *} \\
(10,22)\end{array}$ \\
\hline \multicolumn{6}{|l|}{ Variables de control } \\
\hline LOGRENDOM & $\begin{array}{l}\mathbf{0 , 7 5 *} \\
(8,63)\end{array}$ & $\begin{array}{l}\mathbf{0 , 0 0 8} \\
(0,25)\end{array}$ & $\begin{array}{c}\mathbf{0 , 0 9 4 *} \\
(2,86)\end{array}$ & $\begin{array}{l}\mathbf{0 , 0 1 1} \\
(0,30)\end{array}$ & $\begin{array}{l}\mathbf{0 , 0 7 *} \\
(2,85)\end{array}$ \\
\hline EDUM & $\begin{array}{l}\mathbf{0 , 0 9 *} \\
(4,33)\end{array}$ & $\begin{array}{l}\mathbf{0 , 0 0 5} \\
(0,63)\end{array}$ & $\begin{array}{c}\mathbf{0 , 0 2 1} * \\
(2,63)\end{array}$ & $\begin{array}{c}\mathbf{0 , 0 2 2} * \\
(2,26)\end{array}$ & $\begin{array}{c}\mathbf{0 , 0 7} \\
(0,12)\end{array}$ \\
\hline WHITE & $\begin{array}{l}-\mathbf{0 , 0 5 3} \\
(-0,34)\end{array}$ & $\begin{array}{l}-\mathbf{0 , 0 5 7} \\
(-0,98)\end{array}$ & $\begin{array}{l}\mathbf{0 , 3 2 *} \\
(5,68)\end{array}$ & $\begin{array}{l}\mathbf{0 , 0 1 8} \\
(0,25)\end{array}$ & $\begin{array}{l}-0,27 * \\
(-6,41)\end{array}$ \\
\hline URBAN & $\begin{array}{l}\mathbf{0 , 3 2 *} \\
(1,69)\end{array}$ & $\begin{array}{l}\mathbf{0 , 0 5 5} \\
(0,76)\end{array}$ & $\begin{array}{l}\mathbf{0 , 0 7 9} \\
(1,15)\end{array}$ & $\begin{array}{l}\mathbf{0 , 2 1 *} \\
(2,20)\end{array}$ & $\begin{array}{l}-\mathbf{0 , 0 4 8} \\
(-0,92)\end{array}$ \\
\hline NORTHEAST & $\begin{array}{l}-\mathbf{0}, 37^{*} \\
(-2,39)\end{array}$ & $\begin{array}{l}\mathbf{0 , 1 6 *} \\
(2,78)\end{array}$ & $\begin{array}{l}\mathbf{0 , 1 9 *} \\
(3,35)\end{array}$ & $\begin{array}{l}\mathbf{0 , 0 8 8} \\
(1,19)\end{array}$ & $\begin{array}{l}-\mathbf{0 , 1 0 *} \\
(-2,33)\end{array}$ \\
\hline AGE & $\begin{array}{c}\mathbf{0 , 9 9 *} \\
(15,98)\end{array}$ & $\begin{array}{l}-\mathbf{0 , 7 7 *} \\
(-2,77)\end{array}$ & $\begin{array}{c}\mathbf{0 , 0 6 3 *} \\
(2,33)\end{array}$ & $\begin{array}{l}-0,77 * \\
(-2,24)\end{array}$ & $\begin{array}{l}-\mathbf{0 , 0 3 *} \\
(-1,65)\end{array}$ \\
\hline AGE2 & $\begin{array}{l}-1,91 * \\
(-10,55)\end{array}$ & $\begin{array}{l}\mathbf{0 , 1 7 *} \\
(2,11)\end{array}$ & $\begin{array}{l}-\mathbf{0}, \mathbf{1 7} * \\
(-2,22)\end{array}$ & $\begin{array}{l}\mathbf{0 , 1 9 *} \\
(2,02)\end{array}$ & $\begin{array}{l}\mathbf{0 , 0 8 7} \\
(1,50)\end{array}$ \\
\hline FEMALE & $\begin{array}{l}-\mathbf{0 , 1 8} \\
(-1,52)\end{array}$ & $\begin{array}{l}\mathbf{0 , 1 2 *} \\
(2,31)\end{array}$ & $\begin{array}{l}-\mathbf{0 , 0 8} \\
(-1,53)\end{array}$ & $\begin{array}{c}\mathbf{0 , 1 0} \\
(1,53)\end{array}$ & $\begin{array}{l}\mathbf{0 , 1 0 *} \\
(2,66)\end{array}$ \\
\hline $\mathbf{F}$ & $142,46^{*}$ & $288,81^{*}$ & $1217,92 *$ & 71,93* & $855,98 *$ \\
\hline $\begin{array}{l}\text { R2 ajustado } \\
\text { (pseudo R2) } \\
\text { Observaciones }\end{array}$ & $\begin{array}{c}0,35 \\
2.559\end{array}$ & $\begin{array}{c}0,09 \\
3.167\end{array}$ & $\begin{array}{c}0,28 \\
3.167\end{array}$ & $\begin{array}{c}0,04 \\
3.167\end{array}$ & $\begin{array}{c}0,11 \\
3.167\end{array}$ \\
\hline
\end{tabular}

Nota: Muestra de hijos entre 8 y 30 años. Métodos de estimación: Columna 1: Mínimos Cuadrados, consistentes a heterocedasticidad (matriz de covarianzas de White); Columnas 2-4: Estimaciones Probit; Columna 5: Estimación Probit Ordenada. T- estadísticos entre paréntesis. * Significativo al 5\%.

nerará consecuencias importantes sobre la acumulación global de capital humano, que se consideran en la siguiente sección.

\subsection{Estado de salud y educación}

El estado de salud influye sobre la acumulación de capital humano intelectual tanto en términos cualitativos como cuantitativos. El «efecto calidad» es inherente a 
la reducción de las capacidades de aprendizaje de los niños a causa de un estado de salud no favorable. En particular, un niño dotado de un pobre estado de salud tiende a recibir rendimientos inferiores del proceso educativo. Para demostrar la existencia de este efecto se ha analizado la influencia del estado de salud sobre el desempeño educativo. Para evitar los problemas de endogeneidad que típicamente emergen en estos tipos de análisis, se han utilizado variables instrumentales para el estado de salud de los niños. De forma explícita, se han utilizado los instrumentos tradicionalmente usados en literatura, que estaban disponibles en la encuesta y superaron de forma satisfactoria los tests utilizados a tal efecto. Así, para el BMI de los niños se ha utilizado como instrumentos el BMI de madres y padres, la posesión de un seguro privado y el tiempo necesario para llegar al centro de salud. Todas estas variables tienen una fuerte relación con el BMI, que aproxima el estado de salud, al contrario que con la variable dependiente.

Los resultados de las regresiones presentados en la Tabla 3 evidencian que el estado de salud tiene una influencia positiva y estadísticamente significativa sobre la capacidad educativa de los hijos. En otras palabras, un mejor estado de salud se refleja en una mayor capacidad de completar con éxito los años que componen el proceso educativo. Al contrario, estos resultados parecen indicar que los hijos que han recibido en herencia un estado de salud menos favorable tienden a acumular un nivel inferior de capital humano, a paridad de tiempo dedicado a la educación. Las variables de control utilizadas en las regresiones tienen los signos esperados y son significativas. Se observa una fuerte influencia del nivel educativo de los padres, principalmente de la madre sobre la educación de los hijos. La renta familiar y vivir en zona urbana también parecen influir en la misma dirección respecto a la variable dependiente. Por el contrario, la residencia en el nordeste del país y la asistencia a un colegio público (con excepción de la muestra de mujeres) presentan signos negativos.

\subsection{Estado de salud y duración del proceso de aprendizaje}

Como se ha apuntado en la introducción de este trabajo, el estado de salud también afecta a la acumulación de capital humano a través de otro canal. Dado que los rendimientos de la educación son una función positiva del estado de salud de los niños, los padres de hijos menos saludables podrían elegir niveles inferiores de inversión en educación. Para analizar esta cuestión se ha estimado un modelo cuya variable dependiente es la edad a la cual los niños abandonan la escuela para entrar en el mercado de trabajo, como proxy del tiempo dedicado a la educación. Las variables explicativas consideradas son indicadores distintos del estado de salud. Los resultados se presentan en la Tabla 4. Como se puede observar, todos los indicadores de salud explican significativamente la edad de incorporación al mercado laboral. La interpretación de este resultado es la siguiente. Un mejor estado de salud determina mayores rendimientos de la inversión en educación. Esta es una consecuencia del efecto sobre las capacidades cognitivas de los niños. Sin embargo, hay que considerar 
TABLA 3

EFECTOS DEL ESTADO DE SALUD SOBRE LA EDUCACIÓN DE LOS HIJOS

\begin{tabular}{|c|c|c|c|}
\hline $\begin{array}{l}\text { Variable dependiente } \\
\text { Variables }\end{array}$ & Total & $\begin{array}{c}\text { EAC } \\
\text { Hombres }\end{array}$ & Mujeres \\
\hline BMI & $\begin{array}{c}0,093 * \\
(2,93)\end{array}$ & $\begin{array}{c}0,085^{*} \\
(1,96)\end{array}$ & $\begin{array}{c}0,091 * \\
(2,06)\end{array}$ \\
\hline \multicolumn{4}{|l|}{ Variables de control } \\
\hline EDUM & $\begin{array}{l}\mathbf{0 , 2 3}^{*} \\
(6,89)\end{array}$ & $\begin{array}{l}\mathbf{0 , 2 4 *} \\
(5,10)\end{array}$ & $\begin{array}{l}\mathbf{0 , 2 3 *} \\
(4,78)\end{array}$ \\
\hline EDUF & $\begin{array}{l}\mathbf{0 , 1 6 *} \\
(5,21)\end{array}$ & $\begin{array}{l}\mathbf{0 , 1 5 *} \\
(3,50)\end{array}$ & $\begin{array}{l}\mathbf{0 , 1 7 *} \\
(3,92)\end{array}$ \\
\hline LOGPCIN & $\begin{array}{c}\mathbf{0 , 1 1 *} \\
(11,09)\end{array}$ & $\begin{array}{l}\mathbf{0 , 1 0 *} \\
(8,23)\end{array}$ & $\begin{array}{l}\mathbf{0 , 0 9 *} \\
(7,94)\end{array}$ \\
\hline WHITE & $\begin{array}{c}\mathbf{0 , 0 2 9} * \\
(3,60)\end{array}$ & $\begin{array}{c}\mathbf{0 , 0 3 8 *} \\
(3,41)\end{array}$ & $\begin{array}{l}\mathbf{0 , 0 1 8} \\
(1,61)\end{array}$ \\
\hline URBAN & $\begin{array}{c}\mathbf{0 , 0 2 6 *} \\
(2,61)\end{array}$ & $\begin{array}{r}\mathbf{0 , 0 2 3} * \\
(1,71)\end{array}$ & $\begin{array}{c}\mathbf{0 , 0 2 5 *} \\
(1,76)\end{array}$ \\
\hline NORTHEAST & $\begin{array}{c}-\mathbf{0 , 0 3 8} * \\
(-4,52)\end{array}$ & $\begin{array}{c}-\mathbf{0 , 0 3 9} * \\
(-3,31)\end{array}$ & $\begin{array}{l}-\mathbf{0 , 0 3 5 *} \\
(-3,06)\end{array}$ \\
\hline PUBSCHOOL & $\begin{array}{c}-\mathbf{0 , 0 2 3} * \\
(-2,10)\end{array}$ & $\begin{array}{c}-0,039 * \\
(-2,52)\end{array}$ & $\begin{array}{l}-\mathbf{0 , 0 0 2} \\
(-0,18)\end{array}$ \\
\hline AGE & $\begin{array}{l}\mathbf{0 , 0 6 3 *} \\
(16,21)\end{array}$ & $\begin{array}{c}\mathbf{0 , 0 4 9 *} \\
(8,71)\end{array}$ & $\begin{array}{l}\mathbf{0 , 0 8 0 *} \\
(14,95)\end{array}$ \\
\hline AGE2 & $\begin{array}{c}-\mathbf{0 , 1 9 *} \\
(-17,90)\end{array}$ & $\begin{array}{c}-\mathbf{0 , 1 6 *} \\
(-10,19)\end{array}$ & $\begin{array}{l}-\mathbf{0 , 2 4 *} \\
(-15,82)\end{array}$ \\
\hline Test de Hausman & $11,43^{*}$ & $12,25^{*}$ & $10,54 *$ \\
\hline $\mathbf{F}$ & $169,12 *$ & $\mathbf{9 3 , 5 0 *}$ & $84,20 *$ \\
\hline R2 ajustado & $\mathbf{0 , 3 3}$ & 0,34 & 0,35 \\
\hline Observaciones & 3.167 & 1.700 & 1.467 \\
\hline
\end{tabular}

Nota: Muestra de hijos entre 8 y 30 años. Método de estimación: Variables instrumentales (Instrumentos: BMI de los padres; disponer de un seguro privado de salud y el tiempo necesario para llegar al centro de salud más próximo) T- estadísticos entre paréntesis. * Significativo al 5\%.

también el efecto sobre la esperanza de vida. Una esperanza de vida más larga, relacionada con un mejor estado de salud, determina un mayor beneficio del proceso educativo, en términos de ingresos esperados a lo largo de toda la vida. En consecuencia, padres de niños dotados de un estado de salud favorable tienen más incentivos a invertir en educación. Esto determina periodos educativos más largos y el aumento de la edad de incorporación al mercado de trabajo. 
TABLA 4

EFECTOS DEL ESTADO DE SALUD SOBRE LA DURACIÓN

DEL PROCESO EDUCATIVO

\begin{tabular}{|c|c|c|c|c|}
\hline $\begin{array}{l}\text { Variable Dependiente } \\
\text { Variables }\end{array}$ & $\begin{array}{c}\text { AGEW } \\
\mathbf{1}\end{array}$ & $\begin{array}{c}\text { AGEW } \\
\underset{2}{ }\end{array}$ & $\begin{array}{c}\text { AGEW } \\
\mathbf{3}\end{array}$ & $\begin{array}{c}\text { AGEW } \\
4\end{array}$ \\
\hline $\begin{array}{l}\text { BMI } \\
\text { BMI2 } \\
\text { SAHS } \\
\text { CHRONIC } \\
\text { PHEALTH }\end{array}$ & $\begin{array}{c}\mathbf{0 , 9 2 4 5 *} \\
(3,81) \\
-\mathbf{0 , 0 1 7 7 ^ { * }} \\
(-3,36)\end{array}$ & $\begin{array}{c}-\mathbf{0 , 2 3 6 2 *} \\
(-2,22)\end{array}$ & $\begin{array}{c}-\mathbf{1 , 3 9 4 8 *} \\
(-3,81)\end{array}$ & $\begin{array}{c}-\mathbf{0 , 7 1 4 7 *} \\
(-2,06)\end{array}$ \\
\hline \multicolumn{5}{|l|}{ Variables de control } \\
\hline LOGRENDOM & $\begin{array}{c}\mathbf{1 , 2 5 9 4 *} \\
(10,88)\end{array}$ & $\begin{array}{c}\mathbf{1 , 2 3 1 8 *}^{*} \\
(11,15)\end{array}$ & $\begin{array}{c}\mathbf{1 , 2 6 4 7 ^ { * }} \\
(11,61)\end{array}$ & $\begin{array}{c}\mathbf{1 , 2 4 8 0 *} \\
(11,37)\end{array}$ \\
\hline URBAN & $\begin{array}{c}\mathbf{2 , 5 1 9 8 *} \\
(9,77)\end{array}$ & $\begin{array}{l}\mathbf{2 , 8 5 6 6 *} \\
(11,43)\end{array}$ & $\begin{array}{l}\mathbf{2 , 9 0 9 1 *} \\
(11,70)\end{array}$ & $\begin{aligned} \mathbf{2 , 8 6 8 0 *} \\
(11,48)\end{aligned}$ \\
\hline NORTHEAST & $\begin{array}{c}\mathbf{1 , 0 6 3 5}^{*} \\
(4,97)\end{array}$ & $\begin{array}{c}\mathbf{0 , 8 0 9 6}^{*} \\
(4,01)\end{array}$ & $\begin{array}{c}\mathbf{0 , 8 2 5 2} * \\
(4,10)\end{array}$ & $\begin{array}{c}\mathbf{0 , 8 2 3 8}^{*} \\
(4,07)\end{array}$ \\
\hline MALE & $\begin{array}{l}-1, \mathbf{6 3 0 2} * \\
(-7,76)\end{array}$ & $\begin{array}{c}-\mathbf{1 , 6 6 6 1 *} \\
(-8,30)\end{array}$ & $\begin{array}{c}-1,6448 * \\
(-8,27)\end{array}$ & $\begin{array}{l}-1,6521 * \\
(-8,25)\end{array}$ \\
\hline $\mathbf{F}$ & $80,40 *$ & $101,62^{*}$ & $104,33^{*}$ & $101,44^{*}$ \\
\hline R2 ajustado (pseudo R2) & $\mathbf{0 , 3 0}$ & 0,29 & 0,29 & 0,29 \\
\hline Observaciones & 1.078 & 1.229 & 1.229 & 1.229 \\
\hline
\end{tabular}

Nota: Muestra de hijos entre 8 y 30 años que han accedido al mercado de trabajo. Método de estimación: Mínimos Cuadrados, consistentes a heterocedasticidad (matriz de covarianzas de White). T- estadísticos entre paréntesis.

* Significativo al $5 \%$.

\section{Conclusiones}

Los resultados obtenidos en los ejercicios empíricos permiten evidenciar dos efectos diferenciados. Por un lado, el estado de salud es un factor transmisible entre generaciones. El modelo estimado a partir de la encuesta de estilos de vida de Brasil demuestra que los hijos de padres dotados de un estado de salud pobre reciben como herencia su condición desfavorable. Esta conclusión resulta muy importante a la hora de entender el papel del estado de salud en la transmisión intergeneracional de la capacidad de generar ingresos. Así, se puede explicar la movilidad de ingresos a través 
del estado de salud si es posible demostrar su influencia sobre la formación de capital humano.

Por otro lado, el análisis presentado en este trabajo subraya la existencia de dos canales de influencia de la salud sobre la educación. En primer lugar, los ejercicios empíricos permiten concluir que la salud de los niños afecta a la capacidad de completar con éxito los años del proceso educativo. En otras palabras, un estado de salud favorable determina la habilidad de obtener mayores beneficios del proceso educativo. Segundo, se ha demostrado que los niños dotados de un estado de salud más favorable se incorporan más tarde al mercado del trabajo, disfrutando de periodos educativos más largos. Este resultado ha sido interpretado como un «efecto cantidad» debido a la influencia de la salud sobre los incentivos a invertir en educación. La reducción en las capacidades de aprendizaje, y los menores beneficios de la educación derivados de más cortas esperanzas de vida, actúan como desincentivos para los padres, que racionalmente eligen invertir menos en la educación de los hijos.

\section{Agradecimientos}

Los autores desean agradecer los comentarios y sugerencias, a una versión preliminar de este trabajo, recibidos en las XXVI Jornadas de Economía de la Salud (Toledo, Mayo 2006), la reunión del 69th Health Economists' Study Group (Center for Health Economics, University of York, Junio 2006) y el International Workshop on poverty and social exclusion dynamics (Vigo, Julio 2006).

\section{Bibilografía}

[1] ALDERMAN, H., BEHERMAN, J.R., LAVY, V., y MENON, R. (2001): «Child Health and School Enrollment: A Longitudinal Analysis», Journal of Human Resources $\mathrm{n}^{\circ}{ }^{\circ}$ 36(1), pp. 185-205.

[2] BECKER, G. y TOMES, N. (1986): «Human Capital and the Rise and Fall of Familias», Journal of Labor Economics n. ${ }^{\circ}$ 4(3), Part 2, pp. S1-S39.

[3] BEHERMAN, J. (1993): «The Economic Rationale for Investing in Nutrition in Developing Countries», World Development n. ${ }^{\circ}$ 21, pp. 1749-1771.

[4] BEHERMAN, J. y DEOLALIKAR, A. (1998): «Health and Nutrition» en: Handbook of Development Economics, Vol. 1, H. Chenery and T.N. Srinivasan (eds). New York: North Holland, pp. 631-711.

[5] CASE, A., FERTIG, A. y PAXSON, C. (2005): «The lasting Impact of Childhood Health and Circunstance». Journal of Health Economics n. ${ }^{\circ}$ 24, pp. 365-389.

[6] CASE, A., LUBOTSKY D. y PAXON, C. (2002): «Economic Status and Health in Childhood: The Origins of the Gradient». The American Economic Review n. ${ }^{\circ}$ 92(5), pp. 1308-1334.

[7] CURRIE, J. y MORETTI, E. (2005): Biology as Destiny? Short and Long-Run Determinants of Intergenerational Transmission of Birth Weight. California Center for 
Population Research On-Line Working Paper Series. Los Angeles: University of California.

[8] CURRAIS, L., y RIVERA, B. (2005): «Individual returns to health in Brazil: A Quantile Regression Analysis» en: Health and Economic Growth: Findings and Policy Implications, Guillem López-Casasnovas, Berta Rivera and Luis Currais (eds.), Cambridge, MIT Press.

[9] GLEWWE, P, JACOBY, H. y KING, E.M. (2001): «Early Childhood Nutrition and Academic Achievement: A Longitudinal Analysis» Journal of Public Economics n. ${ }^{\circ}$ 81, pp. 345-68.

[10] INSTITUTO BRASILEIRO DE GEOGRAFÍA E ESTADÍSTICA, IBGE (2003): Pesquisa sobre Padroes de Vida 1996-1997. Rio de Janeiro: IBGE.

[11] MICRONUTIENT INITIATIVE y UNITED NATION CHILDREN'S FUND (2004): Vitamin and Mineral Deficiency Reports. Disponible en: http://www.micronutrient.org/ reports/report.asp?cntry=Full

[12] MIGUEL, E. (2005): Health, Education and Economic Development. in Health and Economic Growth: Findings and Policy Implications, Guillem López-Casasnovas, Berta Rivera y Luis Currais (Eds.), Cambridge: MIT Press.

[13] SAHN, D.E., y Alderman, H. (1988): «The Effects of Variables of Human Capital on Wages, and the Determinants of Labor Supply in a Developing Country» Journal of Development Economics n. ${ }^{\circ}$ 29(2), pp. 157-183.

[14] THE WORLD FOOD PROGRAMME (2006): World Hunger Series 2006: Hunger and Learning. United Nations: World Food Programme, and Stanford University Press. 
